\title{
Exergy Analysis of Porous Medium Combustion Engine Cycle
}

\author{
N. Ravi Kumar \\ Department of Mechanical Engineering, MVGR College of Engineering, Vizianagaram 535005, India \\ Correspondence should be addressed to N. Ravi Kumar, ravi_naradasu@yahoo.com
}

Received 17 July 2011; Accepted 14 August 2011

Academic Editors: C. Del Vecchio and C. F. Gao

Copyright ( $) 2011$ N. Ravi Kumar. This is an open access article distributed under the Creative Commons Attribution License, which permits unrestricted use, distribution, and reproduction in any medium, provided the original work is properly cited.

\begin{abstract}
The need of the fossil fuels is ever increasing in the areas of manufacturing, transportation, heating, and electricity. Nearly $90 \%$ of the energy requirement in transport sector is met by combustion of fossil fuels only. Porous media (PM) combustion is an effective method, which can increase the combustion efficiency as well as minimize environmental pollution. The present paper is aimed at thermodynamic analysis of ideal IC engine cycles with porous media combustion. Two practically possible cycles, namely, periodic and permanent contact of gas with porous medium are considered, and the ideal cycle analyses are made. It is found that PM engine with periodic contact is more efficient than permanent contact type. The exergy analysis also reveals that the energy loss due to irreversibilities in the periodic contact type is less than that of the permanent contact type. With the help of model calculations and graphs, the performance of these two cycles is compared and optimal operating conditions are also evaluated and presented along with the suggestions for enhancing the performance of homogeneous PM combustion in IC engines.
\end{abstract}

\section{Introduction}

Combustion is one of the oldest technologies of mankind. It has been used for more than one million years for different purposes. At present, about ninety percent worldwide energy demand is met by combustion of fuels derived from depletion fossil fuel reserves. To cope up with the impending fuel crisis due to the increasing demand and depleting fossil fuel reserves, there is a need to further improve the performance of existing combustion systems and also to reduce the emissions levels to meet the emission norms. Porous medium combustion (PMC) is one such technology that has many advantages over the existing combustion systems. The steadily increasing use of fossil fuel has threatened the sustainability of life on the earth, since combustion of fossil fuel leads to increase in the emission levels in the atmosphere. Hence, to reduce the pollution level, there is always a demand for development and use of modern efficient technology for combustion of fossil fuel. In recent times, many researchers have developed several newer methods for efficient combustion of fossil fuel. Durst and Weclas [1] established that porous media combustion (PMC) is one such effective method, which can increase the system efficiency as well as minimize environmental pollution.
This technology is entirely different from conventional combustion, which is characterized by a free flame, thin reaction zone, and high temperature gradients. It has got several advantages over conventional combustion systems. In conventional combustion device, the entire combustion takes in the gaseous environment, whereas in porous media, combustion takes place in a 3D solid porous matrix having interconnected pores. Compared to conventional combustion device, the combustion efficiency of porous medium combustor is reasonably high. This increase in efficiency is the outcome of better heat transfer through solid-tosolid conduction and high radiation from the heated solid surface. PMC devices can be classified [1] into two categories, namely, the one in which the combustion is fully confined within the pores of the porous structure and the other one in which the combustion takes place over the surface of the porous matrix.

Durst and Weclas [2] proposed a new combustion concept that fulfills the requirement to perform homogeneous combustion in IC engine using porous medium. The thermodynamics of PM engine is explained by considering four theoretical possible cycles, namely, Carnot cycle, ideal constant volume combustion cycle, periodic contact, and permanent contact of gas with PM engine. They have 
identified that the most realistic cycles for pm combustion are periodic contact and permanent contact types. Further, they have explained the operation of the engine with these cycles. However, they have not taken up the detailed thermodynamic analysis to identify the optimum operating parameters to maximize the efficiency of the cycle. Huang et al. [3] studied some of the aspects of different initial preheating conditions, which could affect the initiation and extinction of super adiabatic combustion (SAC) as well as the subsequent combustion modes in a porous medium. Jugjai and Rungsimuntuchart [4] used the heat recirculation system based on the PM technology in combination with swirling central flow burner to improve the efficiency of the domestic gas burners. Jia and Hsieh [5] conducted experiments in the PM heating burners using the liquefied petroleum gas (LPG) under steady state and transient conditions. He reported a particular phenomenon called metastable combustion along with emission of $\mathrm{CO}$ and NOx. Raviraj and Ellzey [6] conducted experiments to understand the transient behavior of fuel rich PMC with the conversion of methane to hydrogen. Rakopoulos and Giakoumis [7] survey the publications available in the literature concerning the application of the second law of thermodynamics to internal combustion engines. They discussed the main results between the first law and second law analysis. Nield [8] done a critical review of the modeling of viscous dissipation in a saturated porous medium, with applications to either forced convection or natural convection. However, it is felt that effective implementation and efficient use of PM combustion in IC engines is possible only when a detailed thermodynamic analysis of the realistic cycle is available which has not been completely done. Thus, the present paper is aimed at identifying the effects of various operating parameters on the efficiency of the different cycles possible for the operation of a porous medium engine. The exergy analysis of the ideal cycle is also taken up to consider the minimization of waste heat through exhaust gases. Both permanent and periodic gas contact type pm engine cycles are analyzed, and results are presented in graphical form, which will be useful in deciding the parameters suitable for practical operation of a porous medium engine.

\section{Model Development}

Homogenization of the combustion process is the basic concept in PM engine through fuel vaporization, mixing, and energy recirculation in a porous medium. Part of the heat energy of the burned gases is transferred to the porous medium. This energy is utilized back in the cylinder for vaporization of liquid fuel. The practical realization of this concept in a more realistic way is possible according to Durst and Weclas [2] only in two ways, one is the periodic contact type and the other one permanent contact type. Periodic contact system requires a porous medium chamber; that is, an open chamber which is thermally isolated from the head walls and the permanent contact type requires an open chamber to be mounted in the cylinder head. Figure 1 shows $\mathrm{P}-\mathrm{V}$ and T-S diagrams of two types of PM engine cycles that are considered for analysis, namely, periodic contact and permanent contact type. 1-2-3-4-5 is the periodic contact cycle and 1-2'-3-4-5 is the permanent contact cycle.

The periodic contact system requires a valve permitting contact between PM chamber and the cylinder volume. The control of PM-chamber gases is permitted through valve timing. Liquid fuel is injected in to PM volume, and time available for this process and for fuel vaporization is assumed very long. The vaporization process is independent of the spray atomization, engine load, or the engine rotational speed. Because the fuel is injected into the compressed gas with a very low oxidant concentration, the resulting mixture in PM cannot ignite, even under high gas/PM temperature. This noncombustible gaseous charge formed in the PM volume is injected back to the cylinder when the PM chamber valve opens, since the pressure in the PM chamber is much higher than the cylinder pressure. This high-pressure gas discharge from the PM chamber to the cylinder generates a highly turbulent flow conditions in the cylinder supporting mixing and homogenization of the cylinder charge. The other processes (exhaust, suction, and compression) continue in the cylinder without any contact with the PM chamber content.

In case of a permanent contact cycle, there is a permanent contact between working gas and PM volume. The PM combustion chamber is mounted in the head, and during intake there is a weak influence of PM heat capacitor on the in-cylinder air. Further, during compression also there is a small amount of air in contact with hot porous medium. The interaction increases as compression continues, and at TDC the whole air is assumed to be in the PM volume. Near TDC, the heat is released into PM volume. The other processes are as same as in periodic cycle.

\section{Analysis}

The exergy method is a rich and powerful tool for analyzing and understanding the processes as well as process optimization. The main purpose of the exergy analysis is identifying the sources of inefficiencies and discovering the cause of imperfection. Further, the exergy analysis is useful in understanding the influence of thermodynamic phenomena on the process effectiveness and helps in comparison of different thermodynamic factors. It is also useful in determination of the most effective ways of improving the analyzed processes [8-10]. The following assumptions are made in the analysis of two cycles: (1) all the processes are ideal, (2) it is a closed cycle, (3) the working medium is air alone, (4) the properties of air remain constant throughout, (5) there is no regeneration of exhaust gases, and (6) the inlet air temperature is assumed as atmospheric condition, that is, $T_{1}=T_{0}$.

3.1. Periodic Contact Cycle. Cycle 1-2-3-4-5 of Figure 1 represents the periodic contact cycle where the gases after complete compression comes in contact with hot porous 


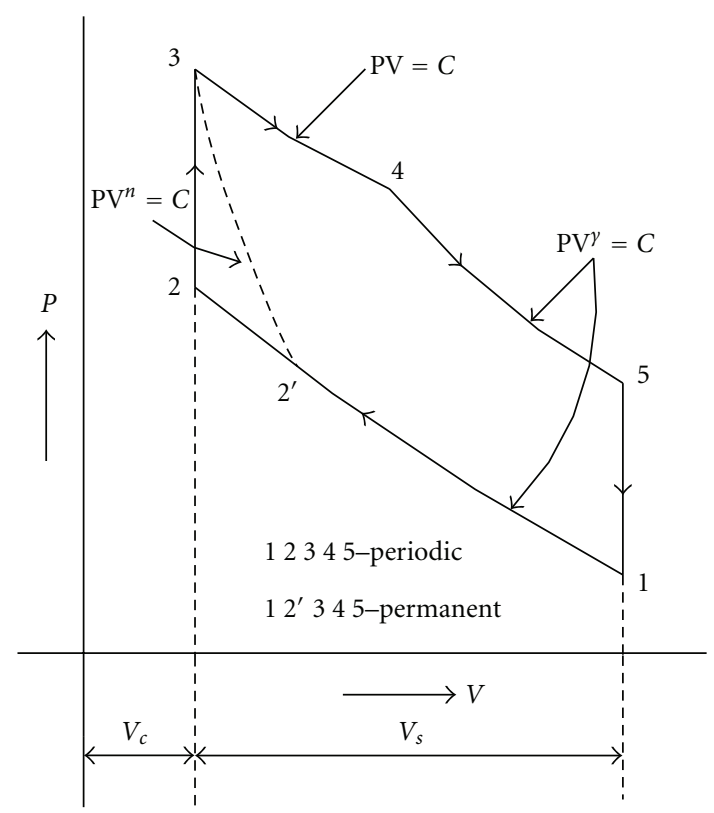

(a) P-V Diagram

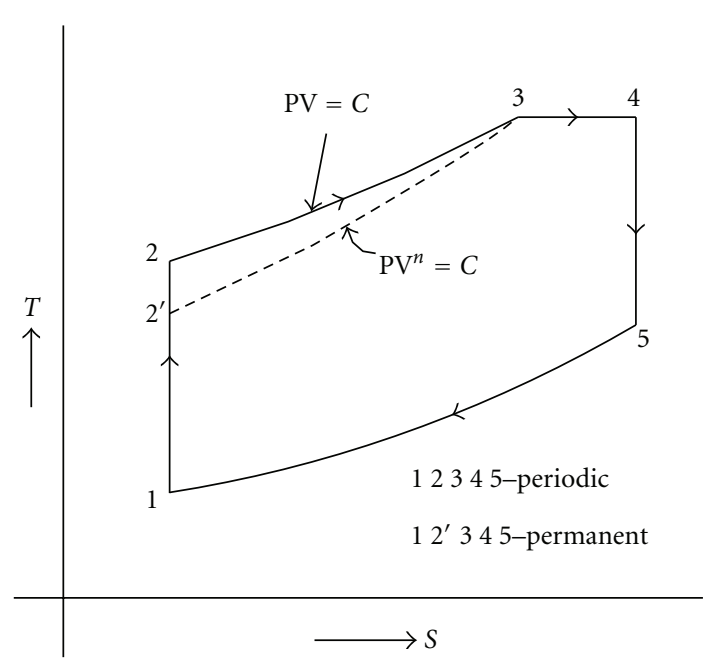

(b) T-S Diagram

FIGURE 1: Ideal cycles for periodic and permanent pm combustion engine.

plug. Gases are ideally compressed from (1) and (2) in which the compression ratio of the cycle is given by

$$
r_{c}=\frac{V_{1}}{V_{2}}=\frac{V_{1}}{V_{3}}=\frac{V_{s}+V_{c}}{V_{c}} .
$$

After compression, the gases are made to come in contact with hot PM chamber during which it is isothermally heated as shown in the process (3) and (4). In diesel and dual cycles, cutoff ratio is defined as the ratio of volumes in a constant pressure process. In the present cycle the volume ratio is considered in isothermal process, hence represented as modified cutoff ratio $\left(\rho^{\prime}\right)$ :

$$
r_{c}=r_{e} * \rho^{\prime}
$$

Thus, the temperature at the end of expansion process can be related to the above parameters as

$$
T_{5}=\frac{T_{4}}{r_{e}^{\gamma-1}}=\frac{\alpha * T_{2}}{r_{e}^{\gamma-1}}=\frac{\alpha * T_{1} * r_{c}^{\gamma-1}}{r_{e}^{\gamma-1}}=\alpha\left(\rho^{\prime}\right)^{\gamma-1} * T_{1} .
$$

Hence, the thermal efficiency of the periodic contact cycle is

$$
\begin{aligned}
\eta_{\text {periodic }} & =1-\frac{C_{v}\left(T_{5}-T_{1}\right)}{R T_{3} \ln \left(V_{4} / V_{3}\right)+C_{v}\left(T_{3}-T_{2}\right)} \\
& =1-\frac{\left(T_{5}-T_{1}\right)}{(\gamma-1) T_{3} \ln \rho^{\prime}+\left(T_{3}-T_{2}\right)} .
\end{aligned}
$$

For the purpose of establishing the second law efficiency, the exergy analysis of the cycle is taken up by considering the irreversibility in all the processes of the cycle 1-2-3-4-5-1 as

$$
\begin{aligned}
& I_{2-3}=T_{0}\left[C_{v} \ln \frac{T_{3}}{T_{2}}+\frac{Q_{3-2}}{T_{3}}\right], \\
& I_{5-1}=T_{0}\left[C_{v} \ln \frac{T_{1}}{T_{5}}+\frac{Q_{5-1}}{T_{1}}\right] .
\end{aligned}
$$

3.2. Permanent Contact Cycle. Cycle 1-2'-3-4-5-1 is the permanent contact cycle as shown in Figure 1, Gases are ideally compressed from (1) and (2):

$$
r_{c}=\frac{V_{1}}{V_{3}}=\frac{V_{1}}{V_{2}^{\prime}} \times \frac{V_{2}^{\prime}}{V_{3}}=r \times r_{\mathrm{AF}},
$$

where $r_{\mathrm{AF}}$ is the adiabatic compression factor:

$$
T_{3}=T_{2}^{\prime}\left(r_{\mathrm{AF}}\right)^{n-1} .
$$

The total heat supplied in the cycle is equal to

$$
\frac{\gamma-n}{n-1} \times m C_{v}\left(T_{2}^{\prime}-T_{3}\right)+m R T_{3} \ln \left(\frac{V_{4}}{V_{3}}\right) .
$$

Thermal efficiency of permanent contact cycle is

$$
\begin{aligned}
& \eta_{\text {permanent }} \\
& \qquad=1-\frac{C_{v}\left(T_{5}-T_{1}\right)}{R T_{3} \ln \left(V_{4} / V_{3}\right)+((\gamma-n) /(n-1)) C_{v}\left(T_{2}^{\prime}-T_{3}\right)} \\
& \quad=1-\frac{\left(T_{5}-T_{1}\right)}{(\gamma-1) T_{3} \ln \left(V_{4} / V_{3}\right)+((\gamma-n) /(n-1))\left(T_{2}^{\prime}-T_{3}\right)}
\end{aligned}
$$




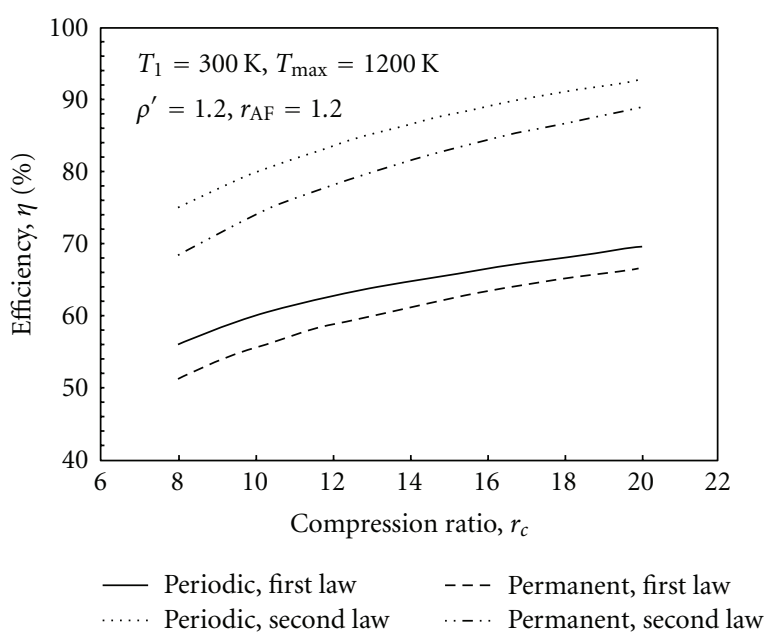

FiguRE 2: Effect of compression ratio on efficiency.

Exergy analysis of the cycle is done by considering the irreversibility in all the process of the permanent contact cycle 1-2'-3-4-5-1.

Irreversibility of the cycle for the process $2^{1}-3$ is given by

$$
I_{2^{\prime}-3}=\left[\frac{\gamma-n}{\gamma-1} \times m R \ln \left(\frac{V_{3}}{V_{2}^{\prime}}\right)-\frac{Q_{2-3}}{T_{3}}\right] T_{0} .
$$

Second law efficiency of the cycle is

$$
\eta_{\mathrm{II}}=\frac{\text { Networkdone }}{\text { Networkdone }+ \text { irreversibilities }} .
$$

\section{Results and Discussion}

The performance of both periodic contact and permanent contact cycles with porous medium is analyzed and compared for a set of operating conditions, and the results are presented through Figures 2 to 9 and are discussed in the following section.

Figure 2 represents the variation of first and second law efficiency values with respect to compression ratio. Both first and second law efficiencies of periodic and permanent cycles are found increasing with increasing compression ratio. At a given compression ratio, the efficiency of periodic cycle is found greater than that of permanent cycle. However, it is found from the figure that with increasing compression ratio the efficiency of permanent cycle approaches that of periodic cycle. Further, the rate of increase in efficiency beyond a compression ratio 20 is marginal in both cases.

Figure 3 shows the effect of maximum temperature on cycle efficiency. With increasing the maximum temperature, the first law efficiency of both cycles appears to be almost constant but actually it is slightly decreased. The periodic cycle efficiency is found greater than that of permanent cycle at a given conditions. The second law efficiency of both cycles is found decreasing with increase in maximum temperature. Hence, from exergy point of view, it is advisable to operate the cycle at lower maximum temperature as this is not going

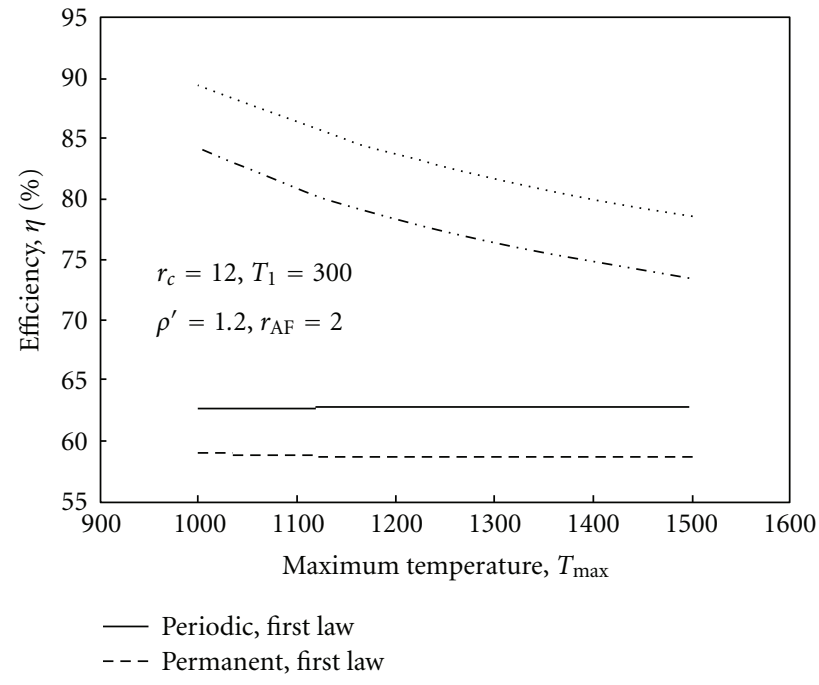

Figure 3: Effect of maximum temperature on efficiency.

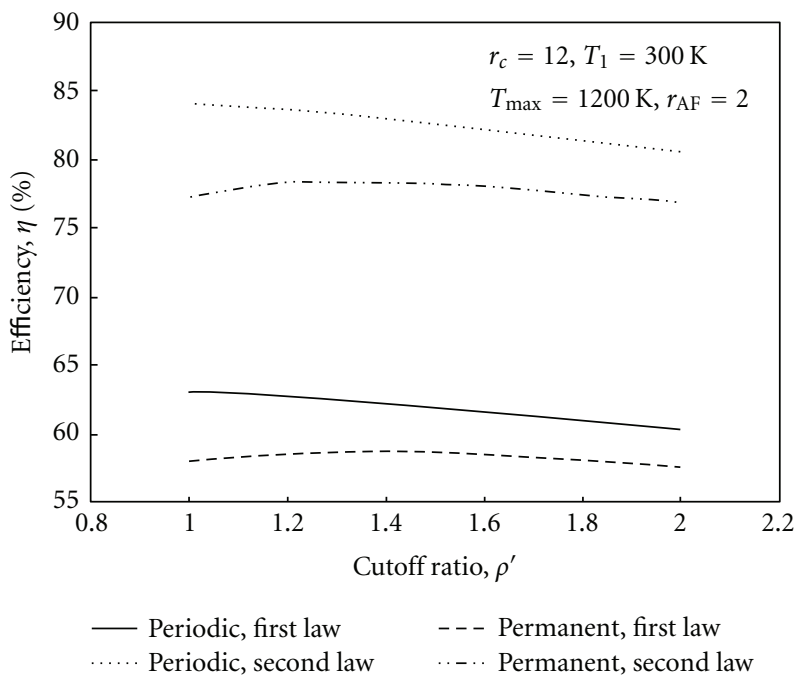

Figure 4: Effect of cutoff ratio on efficiency.

to affect the first law efficiency much and also helps in reducing the NOx formation.

Effect of cutoff ratio on cycle efficiency is shown in Figure 4. It is found from the figure that with increasing cutoff ratio, the first law and second law efficiencies of periodic cycle are constantly decreasing where as for permanent cycle the efficiency increases, reaches a maximum, and then decreases. Approximately at 1.3 cutoff ratio, both the first and second law efficiencies of ideal permanent contact cycle are one found maximum. Figure 5 shows the effect of cutoff ratio on the first law efficiency of a permanent cycle. It is clear from the figure that irrespective of the maximum temperature at around 1.3 cutoff ratio, the efficiency of the cycle is high. Figure 6 shows the effect of maximum temperature on second law efficiency of permanent cycle. The second law efficiency of the permanent cycle is also found maximum when the cutoff ratio is around 1.3. 


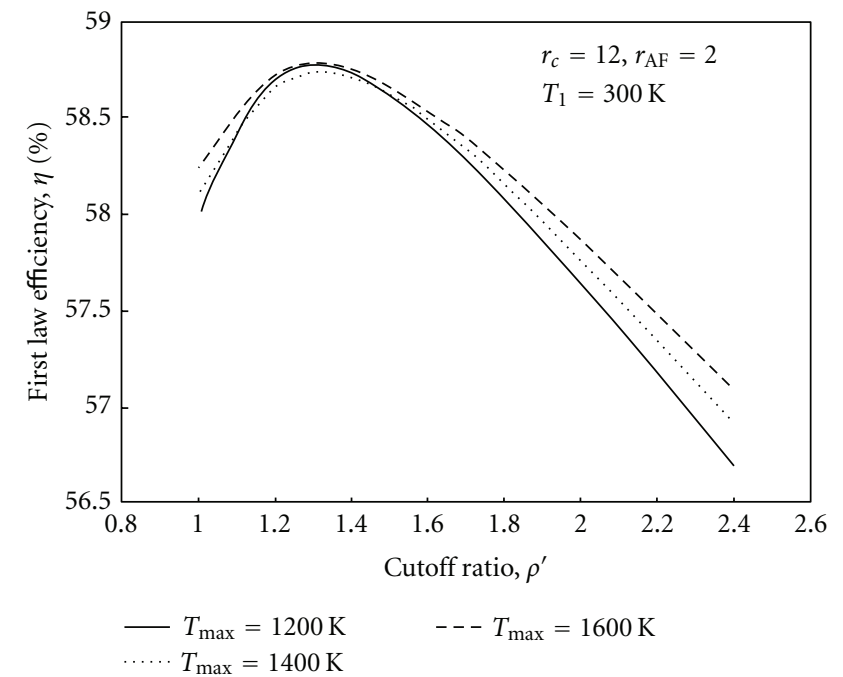

FIGURE 5: Effect of maximum temperature on first law efficiency of permanent cycle.

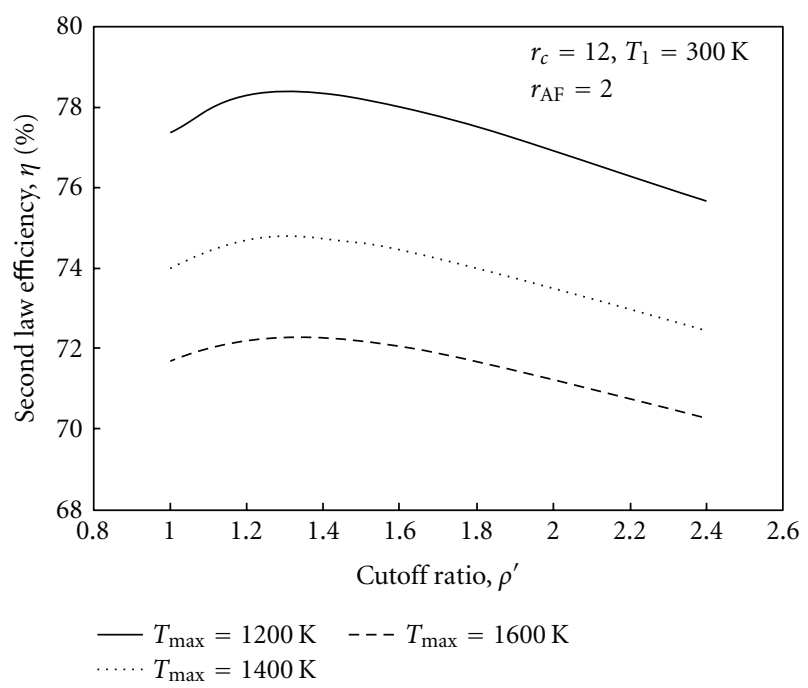

FIGURE 6: Effect of maximum temperature on second law efficiency of permanent cycle.

Figure 7 shows the effect of compression ratio on permanent cycle. With increase in cutoff ratio, the efficiency increases, reaches a maximum, and decreases. The maximum value is found occurring when the cutoff ratio is 1.3.

Figure 8 shows the effect of compression ratio on second law efficiency of the permanent cycle. Irrespective of compression ratio the optimum cutoff ratio found from Figure 8 to develop maximum second law efficiency is around 1.3. Further, it is found that even with different values of adiabatic compression factors, the optimum cutoff ratio is found in the range of 1.2 to 1.4. Hence, it can be concluded that, for best efficiencies, the cutoff ratio in a permanent cycle should be between 1.3 to 1.4 (Table 1).

Figure 9 shows the effect of adiabatic compression factor on permanent cycle. From practical point of view, the
TABLE 1: Effect of modified cutoff ratio on first law efficiency of permanent cycle $\left(r_{c}=12, \mathrm{~T} 1=300 \mathrm{~K}, \mathrm{~T} 3=1200 \mathrm{~K}\right)$.

\begin{tabular}{lcccc}
\hline$\rho^{\prime}$ & \multicolumn{4}{c}{ First Law efficiency $\eta_{1}$} \\
& $r_{\mathrm{AF}}=1.2$ & $r_{\mathrm{AF}}=1.6$ & $r_{\mathrm{AF}}=2$ & $r_{\mathrm{AF}}=2.4$ \\
\hline 1 & 61.68 & 59.62 & 57.97 & 56.59 \\
1.2 & 61.66 & 60.00 & 58.7 & 57.63 \\
1.4 & 61.28 & 59.84 & 58.73 & 57.82 \\
1.6 & 60.77 & 59.47 & 58.48 & 57.67 \\
1.8 & 60.21 & 59.01 & 58.09 & 57.35 \\
2 & 59.63 & 58.51 & 57.65 & 56.95 \\
2.2 & 59.06 & 57.99 & 57.14 & 56.52 \\
2.4 & 58.5 & 57.47 & 56.69 & 56.02 \\
\hline
\end{tabular}

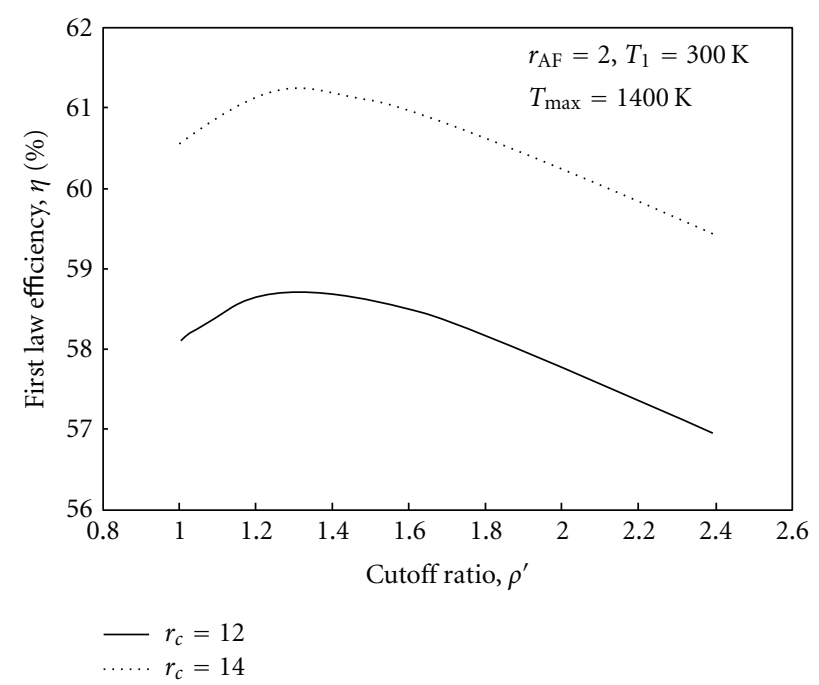

FIGURE 7: Effect of compression ratio on permanent cycle efficiency.

adiabatic compression factor cannot be controlled exactly in a permanent contact cycle. Hence, the effect of variation in the adiabatic compression factor followed by polytropic compression to reach the same maximum temperature is studied by considering different adiabatic compression factors and presenting them in graphical form as shown in Figure 9. It clearly represents that both first law and second law efficiencies decreased with increase in adiabatic compression factors. At AF =1, the efficiency of permanent cycle is equal to that of periodic cycle and with increase in $\mathrm{AF}$ the efficiency goes on decreasing.

\section{Conclusions}

Novel concepts for internal combustion engines based on the application of porous medium technology are discussed. The main attention is focused on analyzing the effect of various operating parameters on two types of PM engines, namely, periodic contact type and permanent type. It is found that the efficiency of periodic contact cycle is more than efficiency of permanent contact cycle in view of both first and second law analysis. Second law analysis reveals 


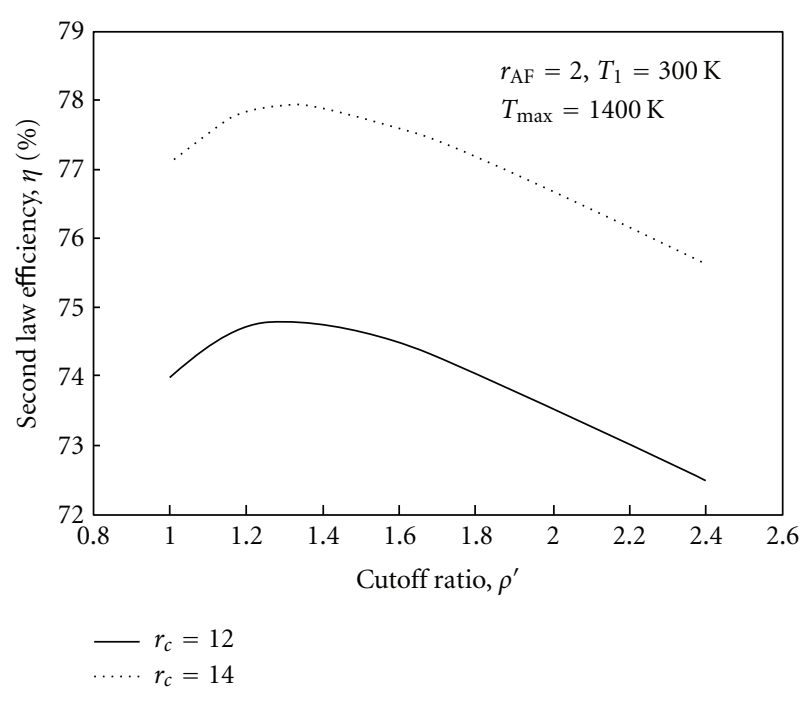

FIGURE 8: Effect of compression ratio on permanent cycle second law efficiency.

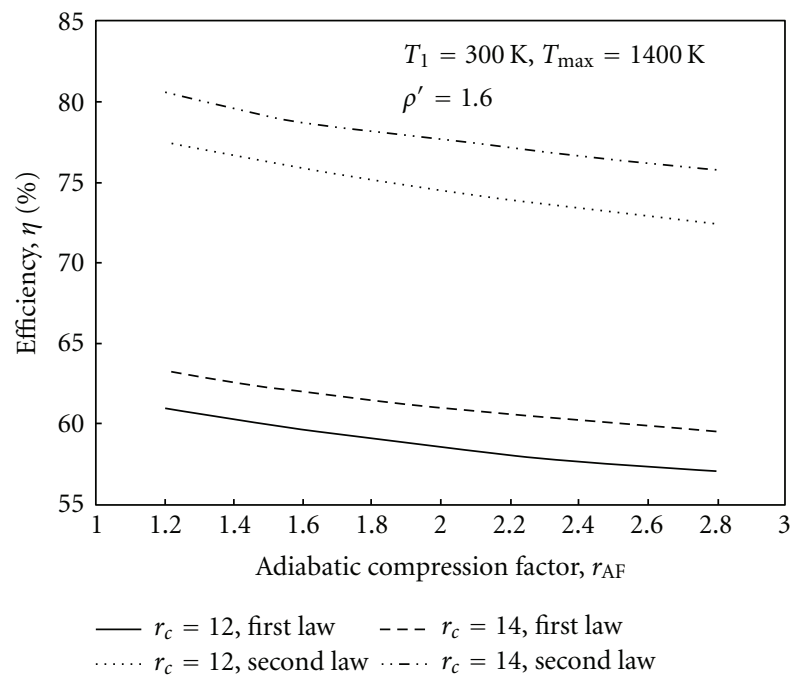

FIGURE 9: Effect of adiabatic compression factor on permanent cycle.

that increase in maximum temperature in the cycle causes decrease in efficiency of the cycle. It is also found that the optimum cutoff ratio of the cycle is in the range of 1.2 to 1.4 and the efficiency of the cycle decreases with increase in adiabatic compression factor.

\section{Nomenclature}

$c_{p}:$ Specific heat, $\mathrm{kJ} / \mathrm{kg} \mathrm{K}$

$c_{v}$ : Calorific value, $\mathrm{kJ} / \mathrm{kg}$

$h$ : Enthalpy, $\mathrm{kJ} / \mathrm{kg}$

I: $\quad$ Irreversibility, $\mathrm{kJ} / \mathrm{kg}$

PM: Porous medium

$r_{c}$ : Compression ratio

s: $\quad$ Entropy, $\mathrm{kJ} / \mathrm{kg} \mathrm{K}$

$T: \quad$ Temperature, $\mathrm{K}$

$V$ : Volume, $\mathrm{m}^{3}$

$w$ : Work done, $\mathrm{kJ} / \mathrm{kg}$.

\section{Suffix}

AF: Adiabatic compression Factor

c: Compression

e: $\quad$ Expansion

$r$ : Rejection

1,2,3 ...: Thermodynamic states in the cycle.

\section{Greek Symbols}

$\eta$ : Efficiency

$\rho^{1}$ : Modified cutoff ratio

$\gamma$ : Ratio of specific heats

$n$ : Polytropic index of compression.

\section{References}

[1] F. Durst and M. Weclas, "A new type of internal combustion engine based on the porous-medium combustion technique," Journal of Automobile Engineering Institution of Mechanical Engineers: Part D, vol. 215, no. 1, pp. 63-81, 2001.

[2] F. Durst and M. Weclas, "A new concept of I.C.Engine with homogeneous combustion in a porous medium," in Proceedings of the 5th International Symposium on Diagnostics and Modeling of Combustion in Internal Combustion Engines (COMODIA '01), Nagoya, Japan, July 2011.

[3] Y. Huang, C. Y. H. Chao, and P. Cheng, "Effects of preheating and operation conditions on combustion in a porous medium," International Journal of Heat and Mass Transfer, vol. 45, no. 21, pp. 4315-4324, 2002.

[4] S. Jugjai and N. Rungsimuntuchart, "High efficiency heatrecirculating domestic gas burners," Experimental Thermal and Fluid Science, vol. 26, no. 5, pp. 581-592, 2002.

[5] F. L. Jia and W. H. Hsieh, "Experimental investigation of combustion in porous heating burners," Combustion and Flame, vol. 138, no. 3, pp. 295-303, 2004.

[6] S. D. Raviraj and J. L. Ellzey, "Numerical and experimental study of the conversion of methane to hydrogen in a porous media reactor," Combustion and Flame, vol. 144, no. 4, pp. 698-709, 2006.

[7] C. D. Rakopoulos and E. G. Giakoumis, "Second-law analyses applied to internal combustion engines operation," Progress in Energy and Combustion Science, vol. 32, no. 1, pp. 2-47, 2006.

[8] D. A. Nield, "The modeling of viscous dissipation in a saturated porous medium," Journal of Heat Transfer, vol. 129, no. 10, pp. 1459-1463, 2007.

[9] V. K. Pantangi and S. C. Mishra, "Combustion of gaseous hydrocarbon fuels within porous media- a review," in Proceedings of the National Conference on Advances in Energy Research (AER '06), pp. 455-461, 2006.

[10] T. Kotas, The Exergy Method of Thermal Plant Analysis, Krieger Publishing Company, Malabar, Fla, USA, 1995. 

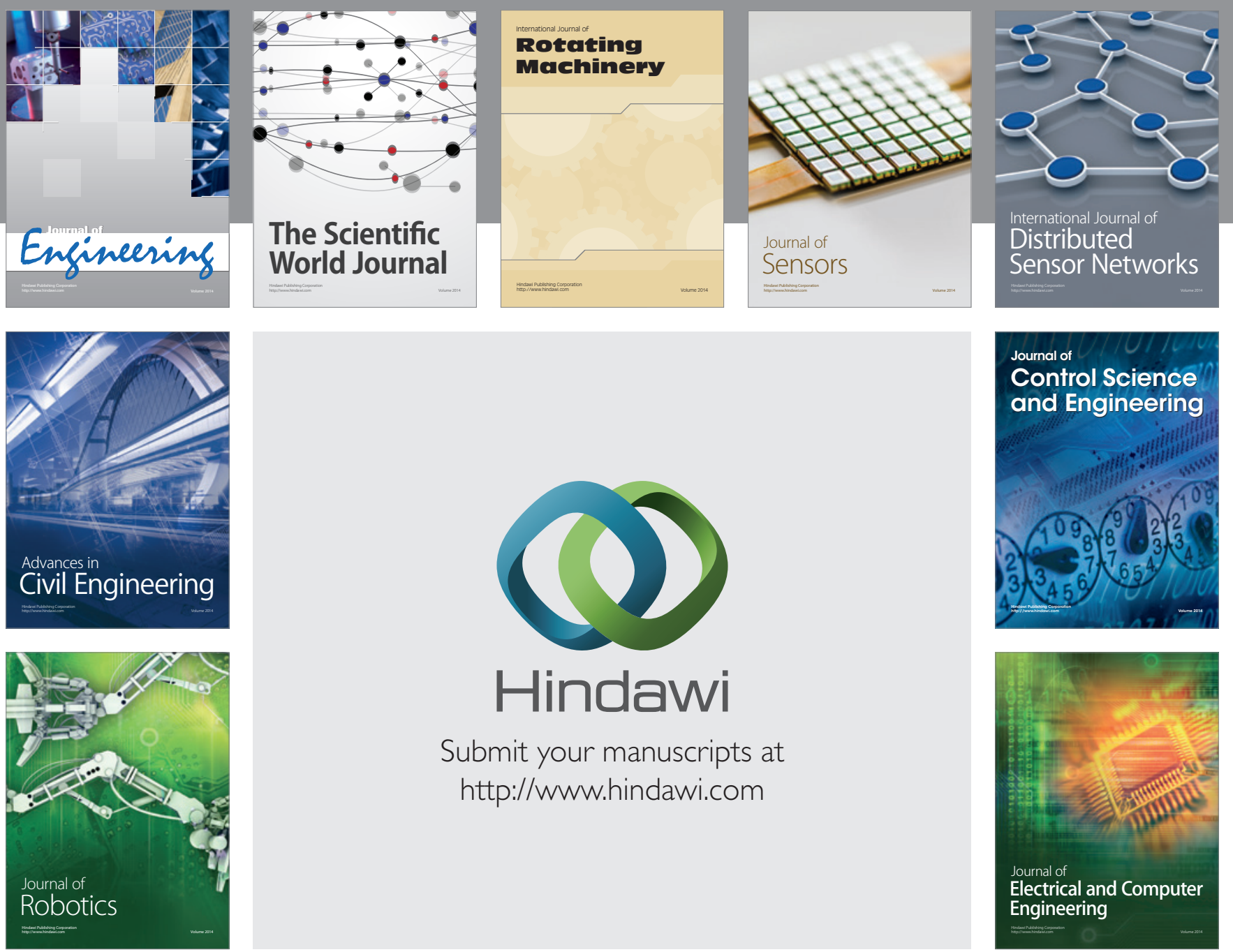

Submit your manuscripts at

http://www.hindawi.com
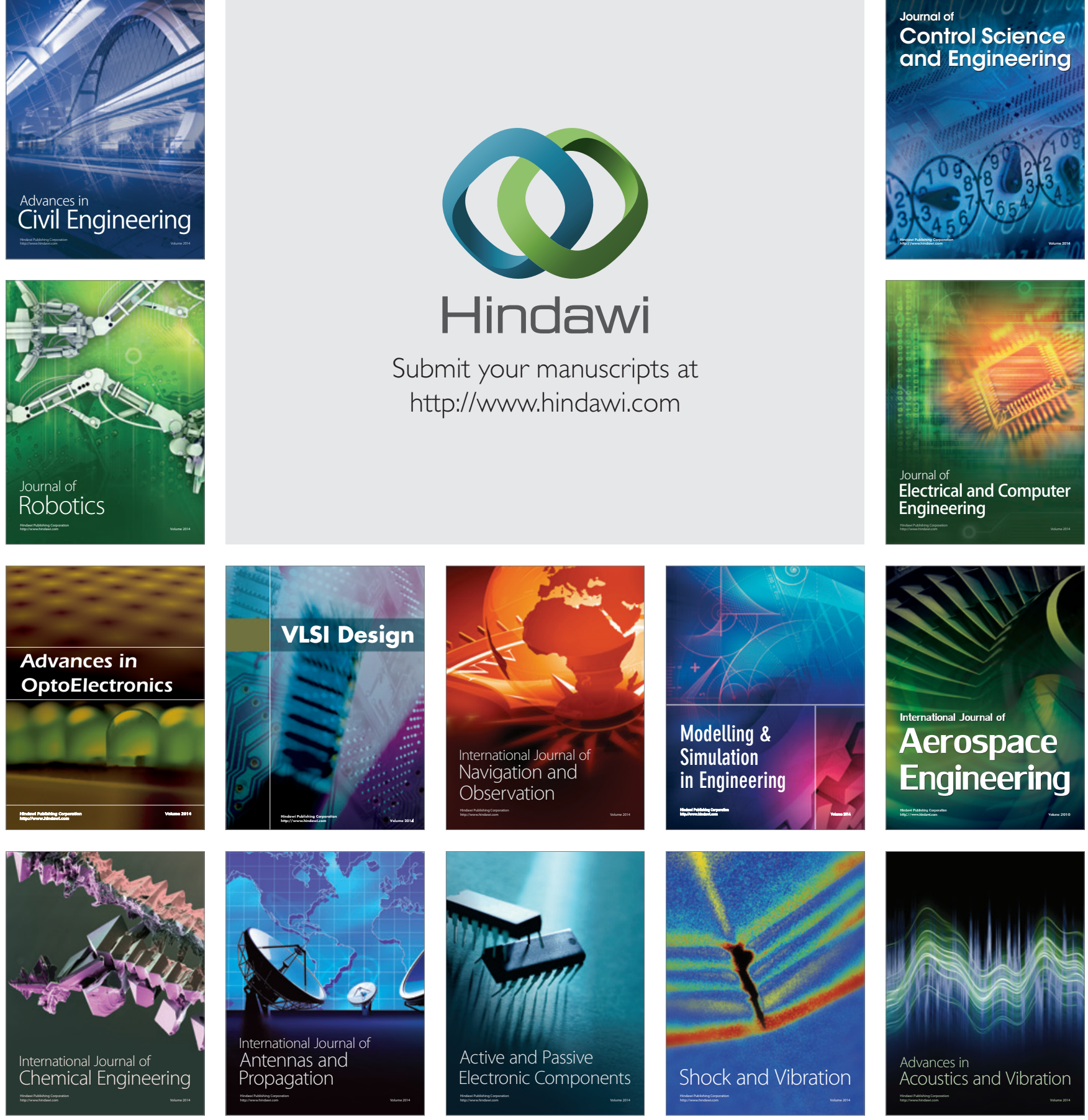